Bol. Acad. peru. leng. 68. 2020 (279-301)

\title{
Morfología del sistema fitonímico medicinal en Tupe (Lima)
}

\author{
Samantha Liz Cruz Ríos \\ Universidad Nacional Mayor de San Marcos \\ samantha.cruz@unmsm.edu.pe
}

Fecha de recepción: $\quad$ 29/03/2020

Fecha de aceptación: $\quad$ 14/09/2020

\section{Introducción}

El pueblo de Tupe, también llamado «comunidad campesina» por el Ministerio de Cultura, está ubicado en la provincia de Yauyos, a $2840 \mathrm{~m} \mathrm{s.} \mathrm{n.} \mathrm{m.,} \mathrm{en} \mathrm{la}$ zona andina del departamento de Lima, a 240 km de distancia. Según Parodi y MacKay, Tupe «tiene una población de 800 habitantes aproximadamente» (2008, p. 266). En dicho pueblo, al igual que en las comunidades campesinas de Cachuy y Aiza, se habla la lengua originaria jaqaru, perteneciente a la familia lingüística Aru (Ministerio de Cultura, 2019).

El jaqaru, según Ramírez (2010), ha existido en la comunidad tupina desde tiempos remotos, sin embargo, no fue hasta la llegada de la lingüística Martha Hardman en 1958 que fue documentada. Dicha lengua es empleada, 
https://doi.org/10.46744/bapl.202002.013

en su mayoría, por la población adulta para denominar los elementos de su entorno. A pesar de la creación de una Educación Intercultural Bilingüe a nivel primario, la lengua no es usada por las poblaciones jóvenes. Esto se ve reflejado en el sistema fitonímico de Tupe, el cual ha sufrido, por el contacto con la lengua castellana, cambios en su composición.

Para este artículo, se considera como antecedente directo la tesis doctoral El sistema fitonímico de los pueblos de Tupe y Cachuy: estudio etnolingüístico, de Emérita Escobar (2017), en el que se describe el sistema fitonímico más específicamente, 180 plantas - de dichas comunidades campesinas, es decir, la ortografía y pronunciación, la caracterización morfológica, el análisis semántico y los usos de las plantas. Asimismo, es pertinente señalar la relevancia de la tesis de licenciatura Hacia una teoría de la complejidad: estudio etnolingüístico y cognitivo de la correlación entre los platos típicos tupinos y su construcción lexical en la lengua jaqaru, de Marco Lovón (2009), debido a que brinda datos importantes sobre la lengua jaqaru, la cual posee la disyuntiva de si ser considerada lengua o dialecto, así como aspectos de la morfología de la palabra y el contacto que existe entre dicha lengua y el castellano. Finalmente, en el aspecto de la motivación semántica, se considera el «Estudio lingüístico de la fitonimia vulgar de las orquídeas en Cuba», de Pedro López (2012), un artículo que hace hincapié en la motivación semántica cuando se denomina a las diferentes variedades de orquídeas que existen en Cuba, entre las cuales destaca la forma y el color de la flor.

La importancia del estudio radica en el carácter botánico de la investigación. Si bien existen estudios previos que ya han descrito con mayor precisión el aspecto lingüístico de los fitónimos en Tupe, estos requerían de conocimiento sobre aspectos botánicos para tener certeza de qué especie se trataba y si esta tenía alguna relación en la denominación de las plantas. Este artículo cuenta con el respaldo de botánicos que han determinado la especie y nombre científico de las 13 plantas de uso medicinal que aquí se analizan.

El artículo se encuentra dividido en siete subacápites principales: la introducción, en donde se detallan aspectos tales como la ubicación de la comunidad campesina de Tupe, la lengua jaqaru, los antecedentes 
pertinentes para el estudio y la importancia que este posee; el marco teórico, el cual se haya compuesto por la terminología empleada a lo largo del artículo; la metodología, donde se detalla el tipo de investigación realizado así como los pasos a seguir que se emplearon para analizar; el análisis, el cual se haya dividido en palabras simples, compuestas y reduplicadas; las conclusiones y las referencias bibliográficas.

\section{Marco teórico}

\subsection{Onomástica}

La onomástica, según Romaní (2004), citando a Dubois (1986), es la «rama de la lexicología que estudia el origen de los nombres propios» (p. 43). Asimismo, Solís (2007) señala que la onomástica se encarga de estudiar los nombres de los lugares (toponimia) y personas (antroponimia).

\subsubsection{Fitonimia}

Escobar (2017), citando a López (2009), señala lo siguiente sobre la fitonimia, también llamada fitonomástica:

[Es] la rama de la onomástica, y por consiguiente de la de la lingüística, que estudia los nombres de las plantas. Su labor consiste en descifrar dónde, cómo y por qué este o aquel nombre se asigna a una planta. Es decir, la fitonimia es el conjunto de fitónimos o nombres de plantas que son el objeto de estudio de la fitonomástica. (p. 37)

Este mismo autor plantea una clasificación de los fitónimos sobre la base de la motivación para denominar.

\subsubsection{Tipos de fitónimos}

López (2012) señala que los fitónimos «surgen espontáneamente relacionados con diferentes campos de asociación del nombre con la realidad objetiva, por lo que podemos plantear que generalmente los fitónimos vulgares son nombres motivados» (p. 525). Plantea un total de 13 tipos de fitónimos: 


\section{https://doi.org/10.46744/bapl.202002.013}

Figura 1

Clasificación de fitónimos

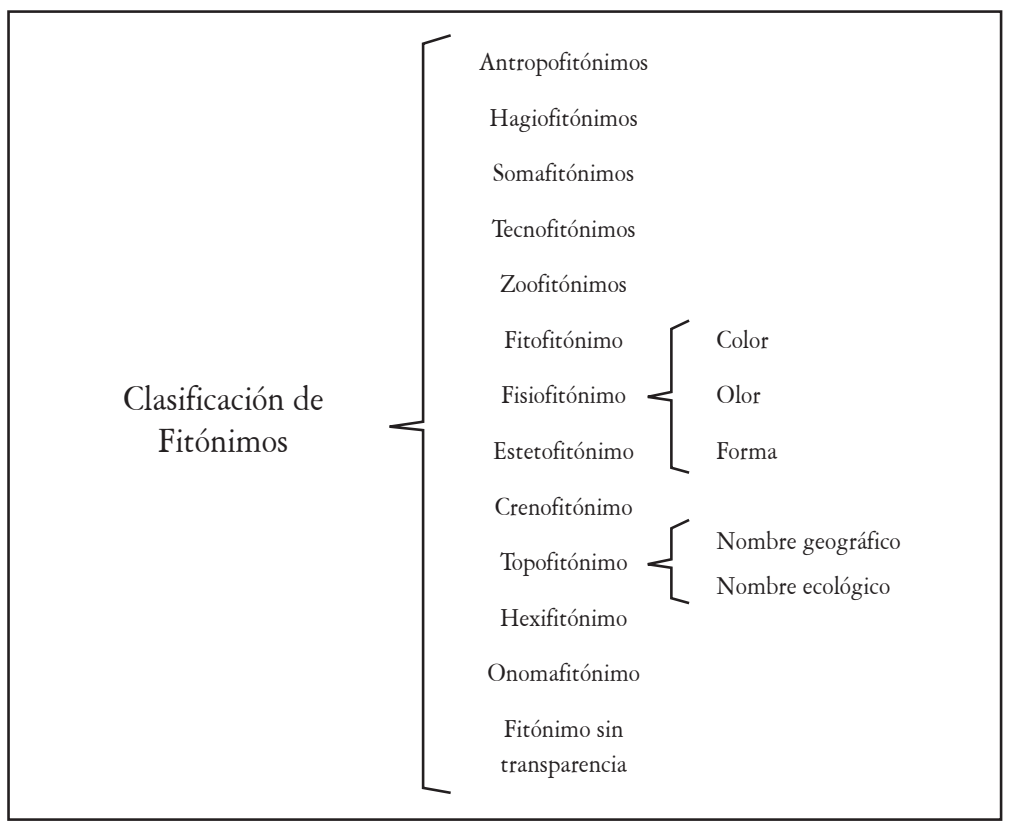

Nota. Esquema basado en la clasificación de fitónimos de López (2012).

\subsection{Morfología}

La morfología es «la parte de la gramática que se ocupa de la estructura de las palabras, las variantes que estas presentan y el papel gramatical que desempeña cada segmento en relación con los demás elementos que las componen» (RAE, 2009, p. 21). Esta morfología se clasifica en la flexión y la formación de palabras. Respecto del proceso que aparece en el estudio, la composición, se define como el «proceso por el que de dos lexemas se crea uno nuevo» (Alonso-Cortés, 2002, p. 223). 
https://doi.org/10.46744/bapl.202002.013

\section{Metodología}

La investigación es de alcance descriptivo, puesto que se encarga de describir la morfología que compone los fitónimos que han sido analizados. Asimismo, el enfoque de la investigación es cualitativo, debido a que se caracterizan los rasgos de los fitónimos.

La muestra analizada en el estudio son los nombres de plantas medicinales del listado de plantas analizadas en la tesis de licenciatura Conocimiento local e importancia del uso de la flora por la comunidad Jaqaru, distrito de Tupe, Lima, de Isabeu Cruz-Ríos. En total, existen 114 fitónimos, de los cuales 86 son plantas medicinales. En el artículo, se analizan 13 fitónimos divididos en palabras simples, compuestas y reduplicadas. Estos 13 fueron seleccionados para evidenciar los procesos que existen entre los 86 fitónimos.

Para el análisis, se emplearon dos diccionarios: el Vocabulario jacarucastellano, castellano-jacaru (aimara tupino), de Neli Belleza, y el Golden Diccionary Portable. Posterior a la búsqueda del origen del fitónimo, se realiza un contraste entre la palabra base y la palabra actual, por lo que se evidencian diversos procesos fonológicos, los cuales son descritos. Finalmente, se determina el tipo de fitónimo que es.

\section{Análisis}

\subsection{Palabras simples}

\subsubsection{Marco}

- Familia: Astaraceae

- Nombre científico: Ambrosia arborescens

- Palabra base: Del jaqaru marku

- Tipo: Sin transparencia

- Forma:

\section{MARKU $>$ MARCO}


https://doi.org/10.46744/bapl.202002.013

\section{Procesos fonológico}

- Descenso vocálico: debido al contacto por parte de los hablantes con el castellano, la palabra marku sufre un descenso vocalico a final de palabra: la posterior alta se vuelve posterior media.

Figura 2

Ambrosia arborescens

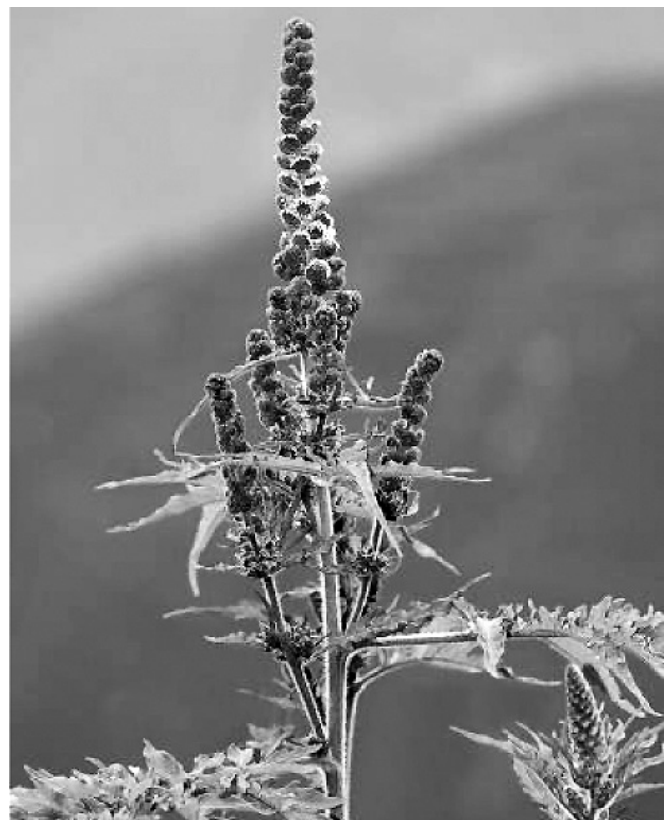

\subsubsection{Matico}

- Familia: Astaraceae

- Nombre científico: Jungia paniculata

- Palabra base: Del jaqaru matiku

- Tipo: Sin transparencia

- Forma: 
https://doi.org/10.46744/bapl.202002.013

\section{MATIKU \MATICO}

\section{Procesos fonológico}

- Descenso vocálico: como en los casos anteriores, el descenso evidenciado es causado por el contacto de lenguas, es decir, el contacto entre el jaqaru y el castellano.

\section{Figura 3}

Jungia paniculata

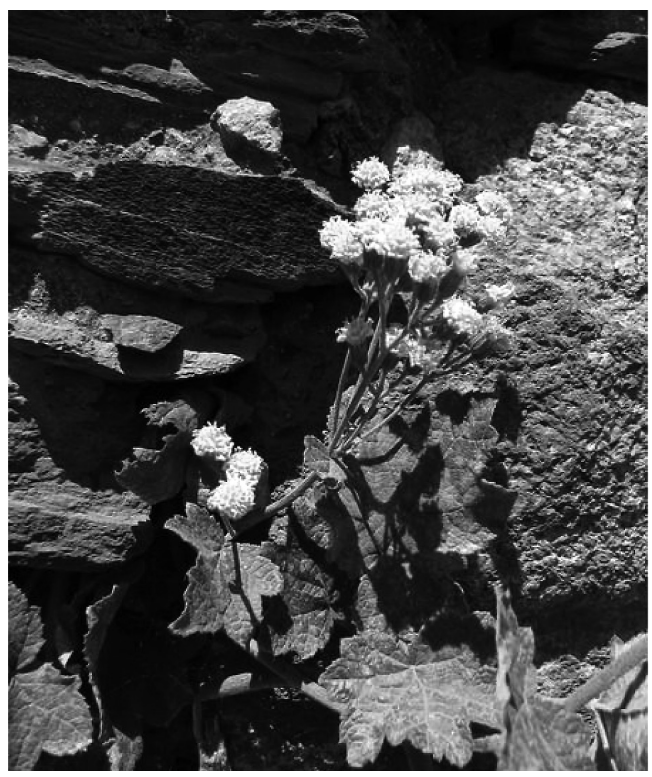

\subsubsection{Pashko}

- Familia: Oxalidaceae

- Nombre científico: Oxalis laxa

- Palabra base: Del jaqaru pashku

- Tipo: Sin transparencia 
https://doi.org/10.46744/bapl.202002.013

\section{- Forma:}

\section{PASHKU $>$ PASHKO}

\section{Procesos fonológico}

- Descenso vocálico: al igual que en los casos anteriores, el descenso vocálico (paso de posterior alta $[\mathrm{u}]$ a posterior media [o]) que se evidencia es producto del contacto con la lengua castellana.

Figura 4

Oxalis laxa

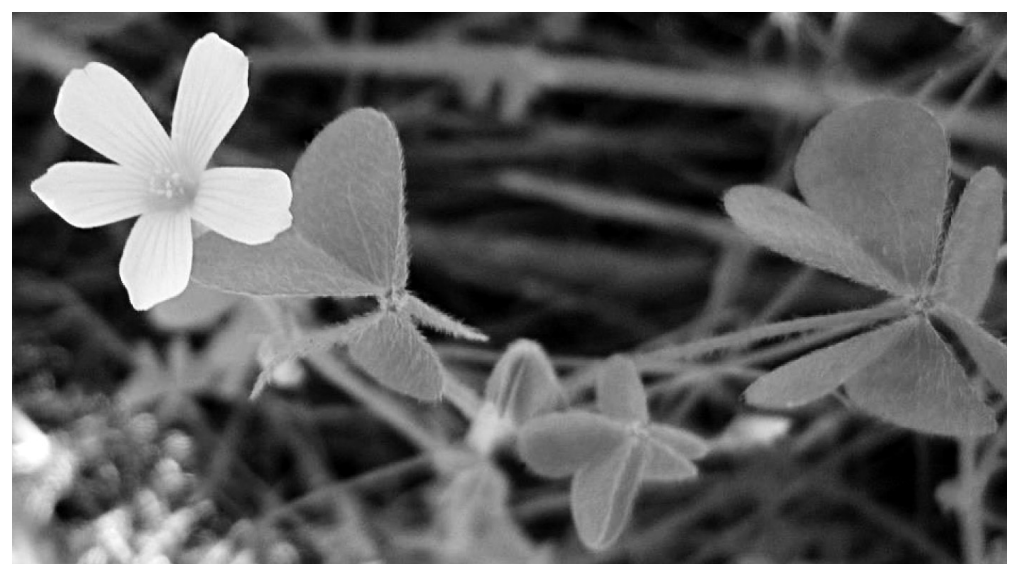

\subsubsection{Pillo}

- Familia: Astaraceae

- Nombre científico: Bidens pilos

- Palabra base: Del jaqaru pillu

- Tipo: Fisiofitónimo

- Forma:

\section{PILLU PILLO}


https://doi.org/10.46744/bapl.202002.013

\section{Procesos fonológico}

- Descenso vocálico: al igual que en los casos anteriores, el descenso vocálico (paso de posterior alta [u] a posterior media [o]) que se evidencia es producto del contacto con la lengua castellana.

\section{Motivación semántica}

El color de la flor (blanco) de la planta es la razón por la que se le denomina pillo 'yerba de flores blancas'.

Figura 5

Bidens pilosa

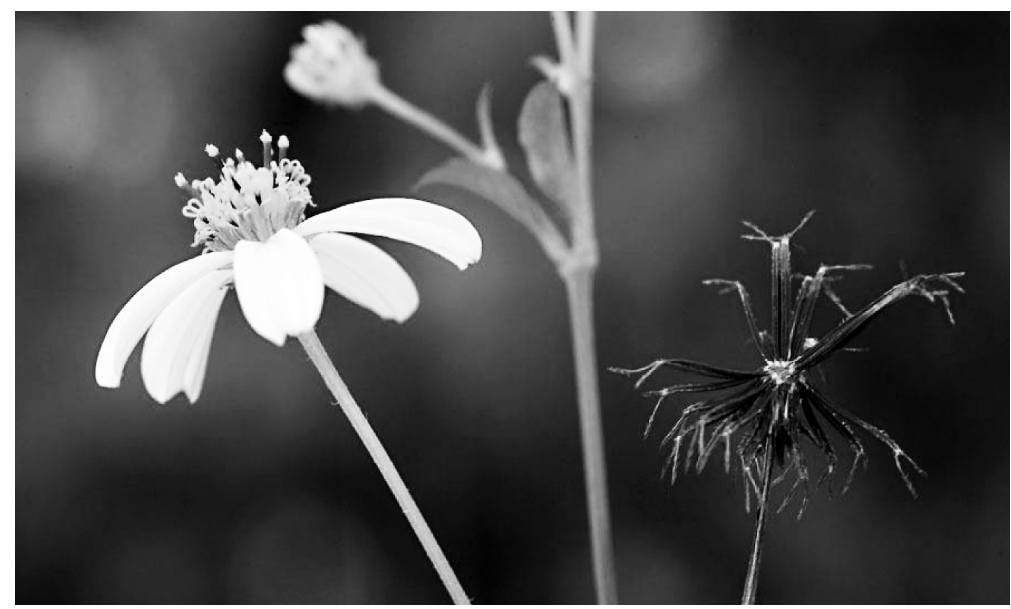

\subsubsection{Pushanko}

- Familia: Astaraceae

- Nombre científico: Xenophyllum poposum

- Palabra base: Del jaqaru pushanku

- Tipo: Sin transparencia

- Forma:

\section{PUSHANKU $>$ PUSHANKO}


https://doi.org/10.46744/bapl.202002.013

\section{Procesos fonológicos}

- Descenso vocálico: como en casos anteriores, se produce un descenso vocálico en el segmento vocálico posterior ubicado a final de palabra.

Figura 6

Xenophyllum poposum

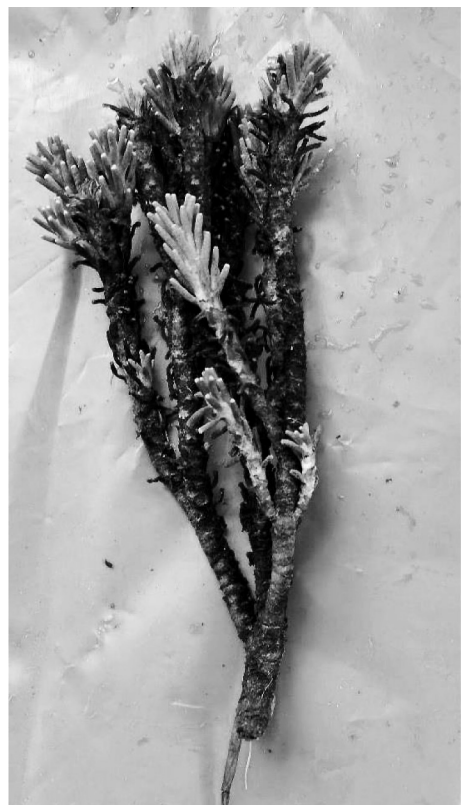

\subsubsection{Sekseka}

- Familia: Boraginaceae

- Nombre científico: Heliotropium incanum

- Palabra base: Del jaqaru sijsaqa

- Tipo: Sin transparencia

- Forma:

\section{SIJSAQA $>$ SEKSEKA}


https://doi.org/10.46744/bapl.202002.013

\section{Procesos fonológicos}

- Descenso vocálico: en este caso, el descenso vocálico ocurre en segmentos a final de palabra, sino en la silaba inicial. Asimismo, estamos frente a un alzamiento de dos segmentos anteriores.

- Espirantización: posterior al descenso, se observa una espirantización, es decir, el paso de una oclusiva a una fricativa con el mismo punto de articulación.

- Alzamiento vocalico: más adelante, se aprecia un curioso alzamiento vocalico: el paso de una baja central [a] a una media anterior [e]. Para explicar este cambio, se puede interpretar de dos maneras: por contacto de lenguas, el sonido más «proximo» a la central baja era la media anterior, u ocurrió una asimilación por la cercanía existente entre estos segmentos vocálicos dentro de la palabra.

- Adelantamiento consonántico: el paso de un sonido posvelar [q] a un sonido velar $[\mathrm{k}]$.

\section{Figura 7}

\section{Heliotropium incanum}

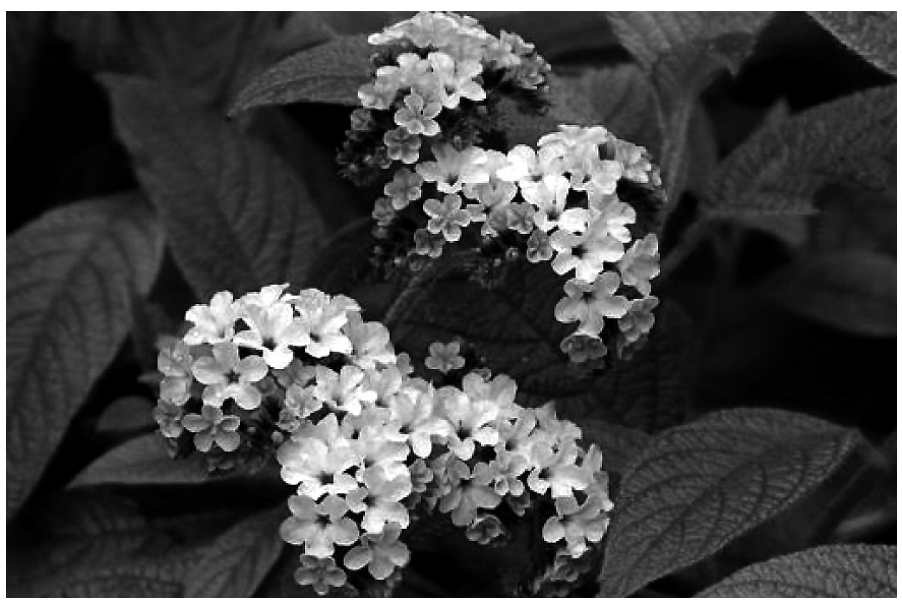


https://doi.org/10.46744/bapl.202002.013

\subsubsection{Talla}

- Familia: Astaraceae

- Nombre científico: Parastrephia quadrangularis

- Palabra base: Del jaqaru taya

- Tipo: Sin transparencia

- Forma:

\section{TAYA TALLA}

\section{Procesos fonológicos}

Alternancia $y / l l$ : si se toma en consideración que los sonidos lateral palatal $[K]$ y semivocal palatal $[y]$ se realizan con una misma pronunciación, es posible señalar un caso de yeísmo, fenómeno que consiste en pronunciar estos dos sonidos como si fuesen uno solo, debido al contacto con el castellano (cuyos hablantes son, en su mayoría, yeístas).

Figura 8

Parastrephia quadrangularis

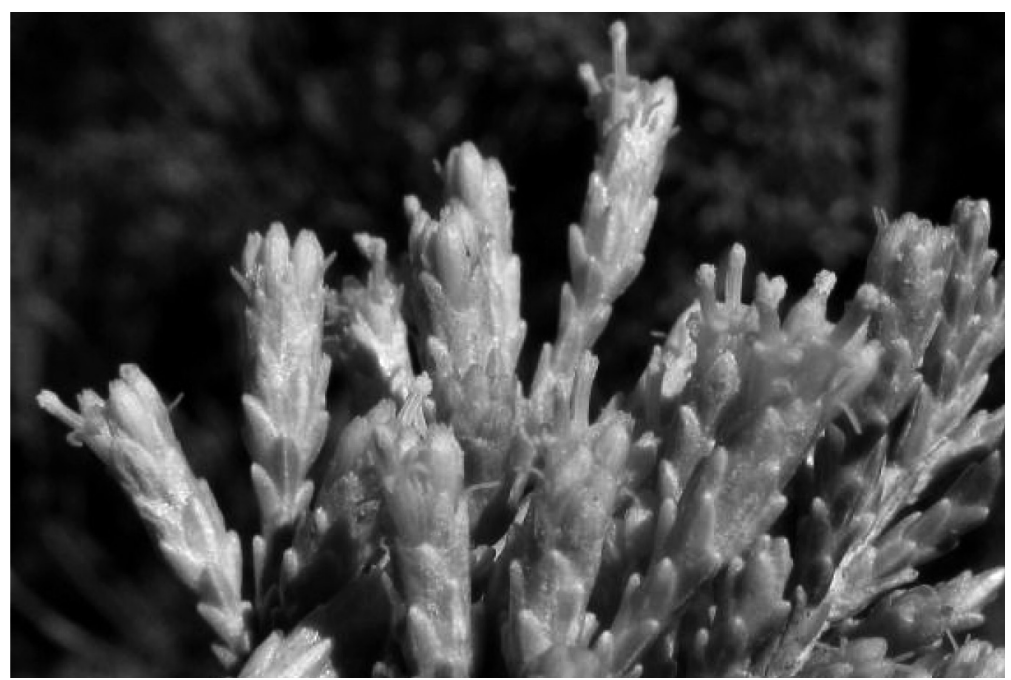


https://doi.org/10.46744/bapl.202002.013

\subsubsection{Wallwa}

- Familia: Fabaceae

- Nombre científico: Otholobium pubescens

- Palabra base: Del vocablo wallwa

- Forma:

\section{WALLWA}

La palabra se mantiene con su forma base, la cual no posee un origen determinado, puesto que wallwa existe en aimara, donde significa 'rastrillo'; en jaqaru, hace referencia a un 'arbusto con flores azules', y en quechua, a una 'planta medicinal'.

Figura 9

Otholobium pubescens

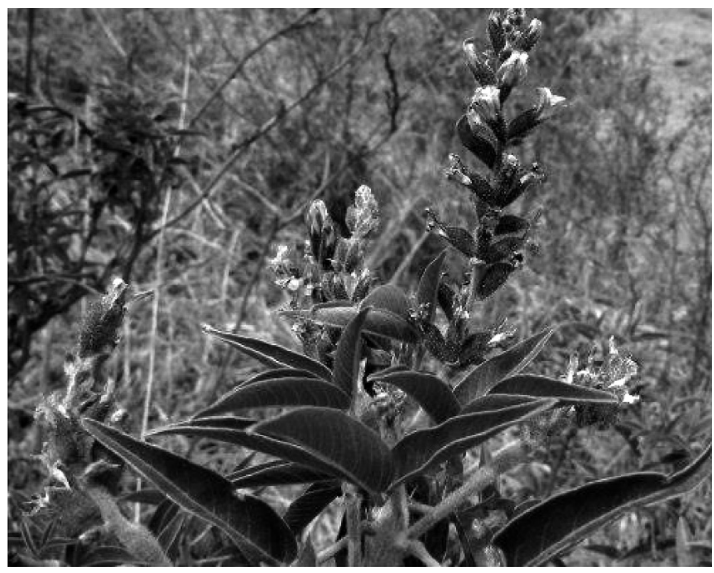

\subsection{Palabras complejas}

\subsubsection{Chillitalla}

- Familia: Astaraceae 
https://doi.org/10.46744/bapl.202002.013

- Nombre científico: Senecio nutans

- Palabra base: Del aimara ch'illi y el jaqaru taya

- Tipo: Sin transparencia

- Forma:

\section{CH`ILLI + TAYA $\boldsymbol{\theta}$ CHILITALLA}

\section{Procesos fonológicos}

- Adelanto de punto de articulación: como se observa, la palabra ha sufrido dos procesos hasta volverse el fitónimo actual. Uno de ellos es el adelantamiento de la lateral, la cual pasó de palatal a alveolar.

- Yeísmo: este fenómeno, producido por el contacto con la lengua castellana, se evidencia en el cambio de la semiconsonante en una lateral.

- Composición: el fitónimo se encuentra compuesto por dos vocablos: la palabra aimara $c b^{\prime}$ illi y el jaqaru taya.

Figura 10

Senecio nutans

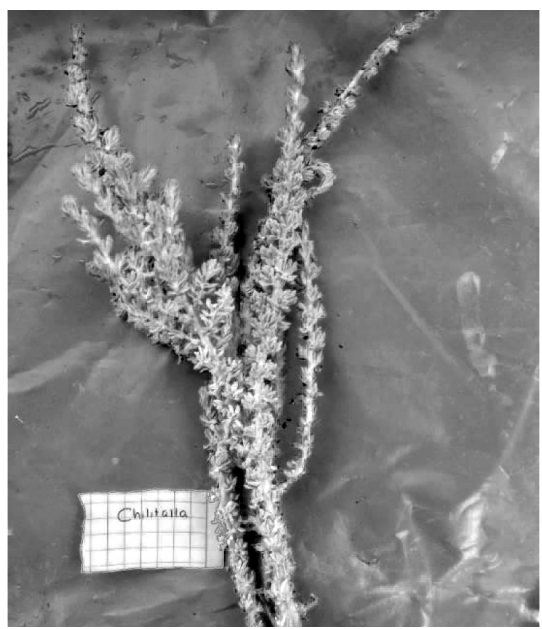


https://doi.org/10.46744/bapl.202002.013

\subsubsection{Pachitallo}

- Familia: Rosaceae

- Nombre científico: Tetraglochin cristatum (Britton) Rotbm

- Palabra base: Del jaqaru pachi 'espina' + castellano tallo

- Tipo: Fisiofitónimo

- Forma:

\section{PACHI $\boldsymbol{y}$ TALLO $\rightleftharpoons$ PACHITALLO}

\section{Procesos morfologico}

- Composición: el fitónimo se encuentra compuesto por el vocablo jaqaru pachi 'espina' y el vocablo castellano tallo, que hace referencia a la parte más larga de una planta.

\section{Motivación semántica}

La razón por la cual se produce esta composición es debido a la forma de la planta. Como se observa en la imagen, la planta esta compuesta por un tallo cubierto de espinas.

Figura 11

Chuquiraga spinosa

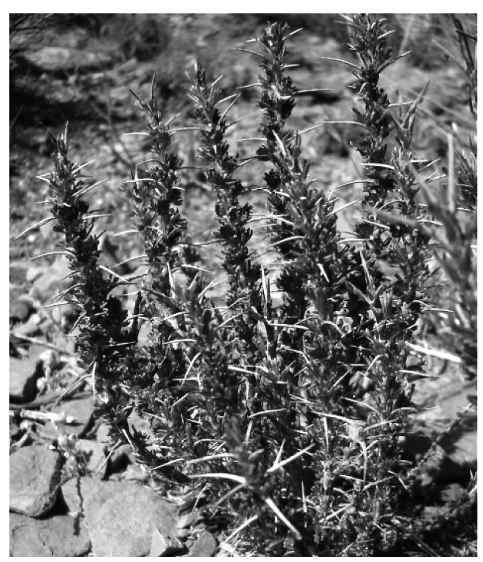


https://doi.org/10.46744/bapl.202002.013

\subsubsection{Wamanchilka}

- Familia: Astaraceae

- Nombre científico: Salvia sagittata

- Palabra base: Del quechua waman y chilka

- Tipo: Fitofitonimia

- Forma:

\section{WAMAN + CHILLKA $\boldsymbol{=}$ WAMANCHILKA}

\section{Procesos fonológicos}

- Adelanto consonántico: como se observa, se produce un adelanto en el punto de articulación: paso de lateral palatal [ $K]$ a lateral alveolar [1], que puede deberse a una busqueda de simplicidad a la hora de pronunciar la palabra.

- Composición: el fitónimo se encuentra compuesto por dos vocablos en quechua, waman, que es halcón, y chilka, que es una planta medicinal.

Figura 12

Salvia sagittata

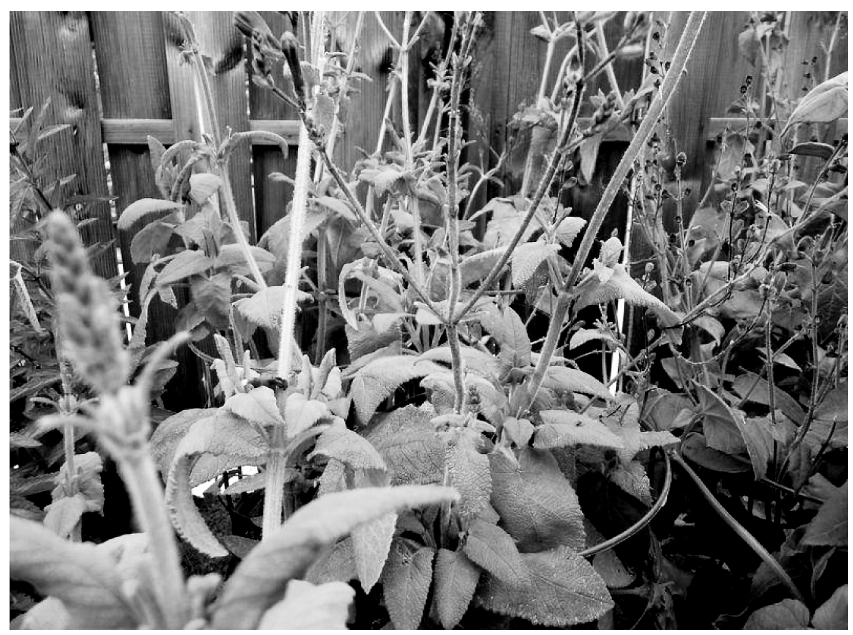




\subsection{Reduplicación}

\subsubsection{Marco Marco}

- Familia: Amaranthaceae

- Nombre científico: Chenopodium petiolare

- Palabra base: Del jaqaru marku

- Tipo: Fitofitónimo

- Forma:

\section{MARKU $\boldsymbol{\nabla}$ MARCO $\boldsymbol{M}$ MARCO MARCO}

\section{Procesos fonológicos}

- Descenso vocálico: el sistema vocálico del jaqaru no presenta la vocal posterior media [o], sin embargo, al castellanizarse el término, se produce un descenso vocalico de la posterior alta $[\mathrm{u}]$ a dicha vocal.

\section{Proceso morfológico y motivación semantica}

Duplicación: Los hablantes poseen dos plantas a las que denominan marco. La razón por la cual ellos repiten dos veces la palabra al referirse al Chenopodium petiolare se debe al tamaño de esta en contraste con el otro marco; es decir, marco y marco marco comparten similitudes en la forma de la flor, pero la segunda es más pequeña que la primera, lo cual es reflejado a través de la duplicación de este vocablo. 
https://doi.org/10.46744/bapl.202002.013

Figura 13

Chenopodium petiolare

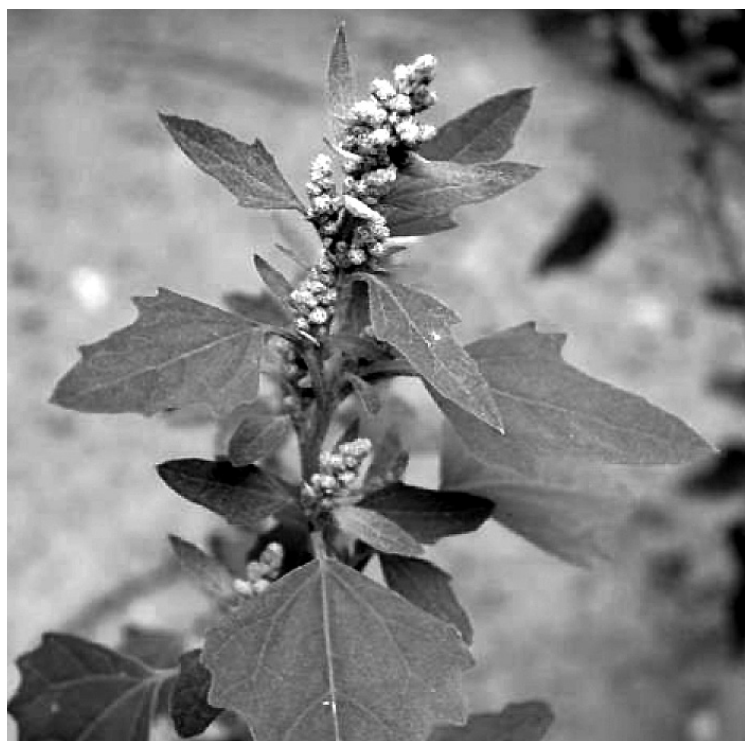

\subsubsection{Wila Wila}

- Familia: Astaraceae

- Nombre científico: Senecio nivalis

- Palabra base: Del vocablo wila

- Tipo: Sin transparencia

- Forma:

\section{WILA \ WILA WILA}

\section{Proceso morfológico}

- Duplicación: como se puede observar, el fitónimo del Senecio nivalis es producto de la duplicación de la palabra wila, cuyo origen es incierto, puesto que la palabra wila significa 'sangre' tanto en jaqaru como en aimara. 
https://doi.org/10.46744/bapl.202002.013

Figura 14

Senecio nivalis

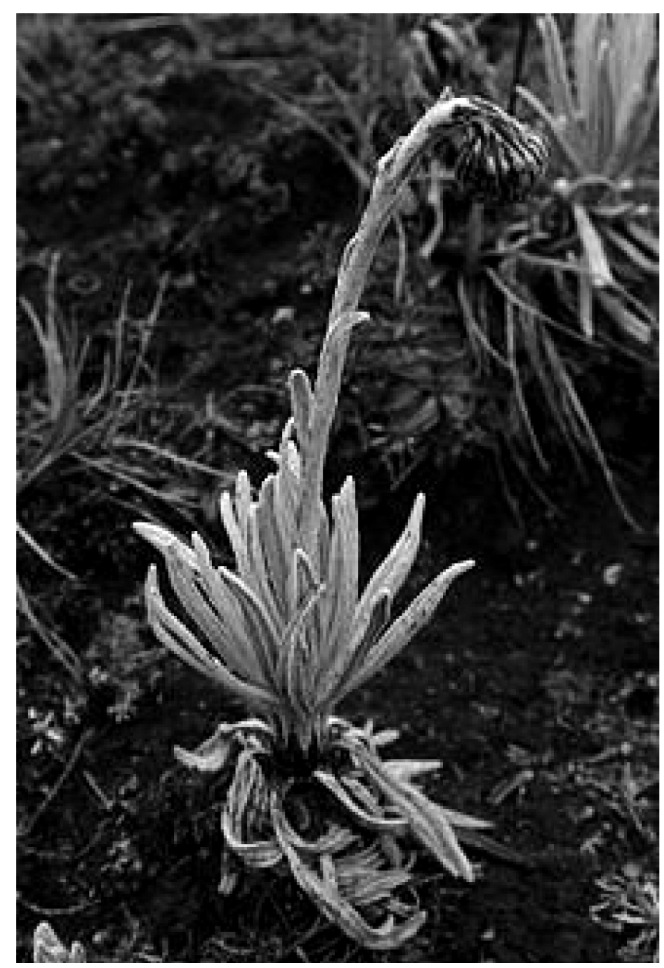

\section{Conclusiones}

El sistema fitonímico medicinal en Tupe tiene características específicas: los fitónimos simples presentan procesos fonológicos tales como el descenso vocálico y la alternancia de las laterales; los fitónimos compuestos, en su mayoría, cuentan con una palabra en jaqaru y otra de un idioma diferente, por lo que se puede especular la presencia de hibridez lingüística; en las palabras reduplicadas, evidenciamos procesos fonológicos, en su mayoría de descenso vocálico. 
https://doi.org/10.46744/bapl.202002.013

En el caso de los tipos de fitónimos, la mayoría son fitónimos sin transparencia, es decir, sin un origen explícito. Asimismo, aparecen casos de fitofitónimos, es decir, que son denominados sobre la base del parecido con otra planta, y casos de fisiofitónimos, los cuales se caracterizan por emplear algunos rasgos de la planta para su denominación. 
https://doi.org/10.46744/bapl.202002.013

\section{BIBLIOGRAFÍA}

ALONSO-CORTÉS, A. (2002). Lingüística. Madrid: Ediciones Cátedra.

Base de Datos Oficial de Pueblos Indígenas u Originarios. (2019). Pueblo

Jaqaru. Lima: Ministerio de Cultura. Recuperado de https:// bdpi.cultura.gob.pe/pueblos/jaqaru

BELLEZA, N. (1993). Vocabulario jaqaru-español, español-jaqaru. Lima, Perú.

ESCOBAR, E. (2017). El sistema fitonímico de los pueblos de Tupe y Cachuy: estudio etnolingüístico. Tesis doctoral. Lima: Universidad Nacional Mayor de San Marcos.

Gobierno Regional del Cuzco. (2005). Diccionario quechua-español-quechua. Cusco: Academia Mayor de la Lengua Quechua.

LOPEZ, P. (2012). Estudio lingüístico de la fitonimia vulgar de las orquídeas en Cuba. Revista de Dialectología y Tradiciones Populares, (2), pp. 515-534.

LOVÓN, M. (2009). Hacia una teoría de la complejidad: estudio etnolingüístico y cognitivo de la correlación entre los platos típicos tupinos y su construcción lexical en la lengua jaqaru.Tesis de licenciatura. Lima: Universidad Nacional Mayor de San Marcos.

OLAECHEA, C. (2011). Creación léxica y morfología en la nomenclatura farmacológica comercial. Tesis de licenciatura. Lima: Universidad Nacional Mayor de San Marcos.

PARODI, L. y MACKAY, M. (2008). Tupe: un pueblo detenido en el tiempo. Arqueologia y Sociedad, (19), pp. 265-292.

RAMÍREZ, W. (2010). Democracia y Participación en espacios rurales. Un estudio en Tupe, una comunidad del Jaqaru en el Perú. Tesis de 
https://doi.org/10.46744/bapl.202002.013

licenciatura. Lima: Universidad Nacional Mayor de San Marcos.

REAL ACADEMIA ESPAÑOLA. (2009). Nueva gramática de la lengua española. Madrid: Espasa.

RENIEC. (2015). Tesoro de nombres jaqaru. Lima: RENIEC.

ROMÁN, J. (2012). La flora y su terminología en la provincia de Jaén: fitonimia y dialectología. Tesis doctoral. Madrid: Universidad Nacional de Educación a Distancia.

ROMANÍ, M. (2004). Toponimia en el Gran Pajonal con especial atención a los topónimos de afiliación asháninka. Tesis de licenciatura. Lima: Universidad Nacional Mayor de San Marcos.

SOLÍS, G. (1997). La gente pasa, los nombres quedan. Lima: Lengua y Sociedad. 
Notas

https://doi.org/10.46744/bapl.202002.013

AneXo 1

Cuadro de fitónimos de Plantas medicinales

\begin{tabular}{|c|c|c|}
\hline Nombre CIENTífico & Nombre & Uso \\
\hline $\begin{array}{l}\text { Chenopodium petiolare } \\
\text { Kunth }\end{array}$ & Marco marco (jq) & Dolor de cintura \\
\hline Ambrosia arborescens Mill. & $\operatorname{Marco}(j q)$ & $\begin{array}{l}\text { Resfrío/Ayuda du- } \\
\text { rante el embarazo }\end{array}$ \\
\hline Bidens pilosa L. & Pillo (jq) & Infecciones (flor) \\
\hline $\begin{array}{l}\text { Tetraglochin cristatum } \\
\text { (Britton) Rothm. }\end{array}$ & Pachitallo (jq + cast) & Hemorragias/renal \\
\hline Oxalis laxa Hook. E Am. & Pashko (jq) & Artritis/Purgante \\
\hline $\begin{array}{c}\text { Jungia paniculata (DC.) } \\
\text { A. Gray }\end{array}$ & Matico (jq) & $\begin{array}{c}\text { Infecciones respira- } \\
\text { torias }\end{array}$ \\
\hline $\begin{array}{l}\text { Parastrephia quadrangula- } \\
\text { ris (Meyen) Cabrera }\end{array}$ & Talla (jq) & Bronquitis \\
\hline $\begin{array}{c}\text { Senecio nivalis (Kunth) } \\
\text { Cuatrec. }\end{array}$ & Wila Wila (jq) & Bronquitis/Resfrío \\
\hline Senecio nutans Sch. Bip. & Chilitalla (jq) & $\begin{array}{c}\text { Dolor de estómago/ } \\
\text { Bronquitis }\end{array}$ \\
\hline Senecio violaefolius Cabrera & Wamanripa (jq) & Bronquitis \\
\hline $\begin{array}{c}\text { Senecio como- } \\
\text { sus var. culcitioides(Sch. } \\
\text { Bip.) Cabrera }\end{array}$ & Q'uli (jq) & Tos \\
\hline $\begin{array}{l}\text { Xenophyllum poposum } \\
\text { (Phil.) V. A. Funk }\end{array}$ & Pushanko (jq) & Gastritis/Bronquios \\
\hline $\begin{array}{l}\text { Heliotropium incanum Ruiz } \\
\text { \& Pav. }\end{array}$ & Sekseka (jq) & Dolor de estómago \\
\hline $\begin{array}{l}\text { Otholobium pubescens } \\
\text { (Poir.) J. W. Grimes }\end{array}$ & Wallwa (incierto) & $\begin{array}{l}\text { Resfrío/Dolor de } \\
\text { estómago }\end{array}$ \\
\hline $\begin{array}{l}\text { Minthostachys mollis } \\
\text { (Benth.) Griseb. }\end{array}$ & Muña (incierto) & Gases \\
\hline $\begin{array}{c}\text { Salvia sagittata Ruiz \& } \\
\text { Pav. }\end{array}$ & Wamanchilka (jq) & Dolor de parto \\
\hline
\end{tabular}

